\title{
Calidad nutricional del rastrojo de maca (Lepidium peruvianum Chacón) en cuyes
}

\author{
Nutritional QUality of MACa STUbble (Lepidium peruvianum Chacón) \\ IN GUINEA PIGS \\ Jorge Castro Bedriñana ${ }^{1,2}$, Doris Chirinos Peinado ${ }^{1}$, Jorge Calderón Inga ${ }^{1}$
}

\section{Resumen}

\begin{abstract}
Con el objetivo de evaluar la calidad nutricional del rastrojo de maca (Lepidium peruvianum Chacón) proveniente de la trilla para la colección de la semilla botánica, en la provincia de Junín ( $4000 \mathrm{msnm}$ ), Perú, se determinó la composición química proximal, los coeficientes de digestibilidad, el contenido de nutrientes digestibles totales, el consumo voluntario y la ingesta proteica del rastrojo picado de maca. Se utilizaron 4 cuyes machos de 4 meses de edad de la raza Wanka dispuestos en jaulas metabólicas que permiten colectar heces libres de orina. Los contenidos de humedad (H), materia seca (MS), proteína total (PT), extracto etéreo (EE), fibra cruda (FC), extracto libre de nitrógeno (ELN) y ceniza del rastrojo de maca fueron $11.30,88.70,6.97,3.58,36.35,44.53$ y $8.58 \%$, respectivamente; los coeficientes de digestibilidad de la MS, PT, EE, FC, ELN, materia orgánica (MO) y nutrientes digestibles totales (NDT) del rastrojo de la maca fueron 74.79, 68.00, $75.27,71.78,77.08,74.21$ y $71.21 \%$, respectivamente. Los consumos promedio de rastrojo fresco de maca por cuy/día y como porcentaje del peso vivo fueron $21.46 \mathrm{~g}$ y $3.25 \%$, respectivamente; mientras que el consumo de MS como porcentaje del peso vivo y en gramos por kilogramo de peso metabólico fue de $2.89 \%$ y $146.11 \mathrm{~g}$, respectivamente. La ingesta promedio diaria de proteína por kilogramo de cuy vivo fue de $2 \mathrm{~g}$.
\end{abstract}

Palabras clave: calidad nutricional; rastrojo de maca; digestibilidad; cuyes; nutrientes digestibles totales

\section{AbSTRACT}

The objective of this study was to evaluate the nutritional quality of maca stubbles (Lepidium peruvianum Chacon) from threshing for the collection of botanical seed, in the province of Junín (4000 m above the sea level), Peru. The proximal chemical composition

\footnotetext{
${ }^{1}$ Laboratorio de Nutrición Animal, Facultad de Zootecnia, Universidad Nacional del Centro del Perú, Huancayo, Perú

${ }^{2}$ E-mail: jorgecastrobe@yahoo.com
}

Recibido: 15 de octubre de 2017

Aceptado para publicación: 30 de enero de 2018 
and the digestibility coefficients, the total digestible nutrient contents, the voluntary consumption, and the protein intake of the maca stubbles were determined. Four 4month-old male Wanka guinea pigs were placed in metabolic cages to collect urine-free faeces. The contents of moisture (H), dry matter (DM), total protein (TP), ether extract (EE), crude fibre (CF), nitrogen-free extract (NFE) and ash of maca stubbles were 11.30, $88.70,6.97,3.58,36.35,44.53$ and $8.58 \%$, respectively; the digestibility coefficients of the DM, PT, EE, EC, ELN, organic matter (OM) and total digestible nutrients of maca stubbles were $74.79,68.00,75.27,71.78,77.08,74.21$ and $71.21 \%$, respectively. The average consumptions of fresh maca stubbles per guinea pig/day and as a percentage of live weight were $21.46 \mathrm{~g}$ and $3.25 \%$, respectively; while the consumption of DM as a percentage of live weight and in grams per kilogram of metabolic weight was $2.89 \%$ and $146.11 \mathrm{~g}$, respectively. The average daily protein intake per kilogram of live guinea pig was $2 \mathrm{~g}$.

Key words: nutritional quality; maca stubble; digestibility; guinea pigs; total digestible nutrients

\section{INTRODUCCIÓN}

El rubro alimentación sobrepasa el 65\% del costo de producción animal en diversos sistemas de producción (Shimada, 2007), por lo que todo intento por reducirlo mejorará la rentabilidad del criador. Una forma es a través de la incorporación de ingredientes alimenticios locales y que tengan un bajo costo y adecuada calidad nutricional (Castro y Chirinos, 2017). Una alternativa viable en la alimentación de herbívoros menores es la incorporación de residuos de cosecha, tal como el rastrojo de maca (Lepidium peruvianum Chacón, también conocida como Lepidium meyenii Walp), el cual luego de la trilla y obtención de semilla botánica es desperdiciado, por lo que cabe la posibilidad de utilizarlo en la alimentación animal, previa evaluación de su valor nutritivo.

La maca es una planta peruana herbácea, bianual, de la familia Brassicaceae, que crece exclusivamente en los Andes centrales entre 4000 y $4500 \mathrm{msnm}$ (Gonzales, 2012). La zona agroecológica de este cultivo se ubica en las alturas de los departamentos de Junín y Pasco, principalmente en San Juan de Jarpa y Ahuac en Huancayo; Ondores, Carhuamayo y Junín en Junín; y Ninacaca, Vicco y San Antonio de Rancas en Cerro de
Pasco. El rendimiento promedio de maca, en la región Junín está entre 6.2 y 9.4 t/ha (Perú Biodiverso, 2011). Entre los departamentos de Junín y Pasco se contaban con 30 mil hectáreas en el año 2000, lo cual que representa el $80 \%$ de la producción nacional (BCRP, 2013).

Los hipocótilos de maca son utilizados como suplemento alimenticio y por sus propiedades medicinales (Gonzales, 2006). La evidencia científica experimental ha demostrado que la maca tiene propiedades nutricionales, energizantes, potenciadoras de la fertilidad y antiartríticas (Bianchi, 2003), con gran potencial como adaptógeno y nutracéutico en la prevención de varias enfermedades (Gonzales, 2012), de allí el creciente interés por los productos de la maca en muchas partes del mundo.

La maca secada al aire contiene agua $10.4 \%$, proteína total $10.2 \%$, lípidos $2.2 \%$, ELN $59.0 \%$, fibra cruda $8.5 \%$ y ceniza $4.9 \%$, destacando su perfil de aminoácidos y su riqueza en leucina, fenilalanina, lisina, valina, isoleucina y metionina (Dini et al., 1994). En la fracción lipídica, destaca su contenido de ácido linoleico (32.6\%), ácido palmítico (23.8\%) y ácido oleico (11.1\%). La fracción de esteroles contiene sitosterol (45.5\%), campesterol $(27.3 \%)$, ergosterol (13.6), 
brassicasterol (9.1\%) y AT'22-ergostadienol (4.5\%). Tiene importantes contenidos de hierro, calcio, cobre, magnesio, yodo, zinc, fósforo, potasio y una serie de componentes bioactivos (Dini et al., 2002; Muhammad et al., 2002; Esparza et al., 2015).

Camilonga y Flores (2003), al suplementar cuyes durante 150 días con maca tostada y molida ( $15 \mathrm{~g} /$ día) reportaron un incremento significativo en el número de crías y porcentaje de fertilidad. En ratas se han reportado efectos positivos sobre la espermatogénesis (Gonzales et al., 2006) y efectos antiinflamatorios de la próstata (Gonzales et al., 2005) y en ratones tratados con extracto metalónico se incrementó la producción de óxido nítrico por los macrófagos peritoneales (Alzamora et al., 2007).

En la cosecha de maca se obtiene un rastrojo, compuesto por tallos y hojas, el cual es desperdiciado, no teniéndose referencias sobre su posible uso en la alimentación animal. Los componentes nutricionales y bioactivos están concentrados en la raíz de la maca, aunque diferentes fracciones están presentes en las hojas; así, Li et al. (2001) identificó los aromáticos bencilglucosinolatos (glucotropaeolin) y el p-metoxibencilglucosinolato en hojas frescas y secas. Asimismo, Castañeda et al. (2010) empleando cromatografía líquida de alta presión reportan un contenido de $3.5 \mathrm{mg}$ de quercetina por gramo de muestra en hoja de maca, así como contenidos de ácido palmítico, linoleato de etilo, linolenato de etilo, palmitato de etilo, ácido esteárico y linolenato de metilo. El presente estudio evaluó la calidad nutricional del rastrojo de maca en la alimentación de cuyes.

\section{Materiales y Métodos}

Las fases pre-experimental y experimental de los ensayos de digestibilidad y los análisis de laboratorio se llevaron a cabo en la Granja Agropecuaria de Yauris y el Laboratorio de Nutrición Animal de la Facultad de Zootecnia de la Universidad Nacional del Centro del Perú, respectivamente, ubicados en la provincia de Huancayo, departamento de Junín, Perú, a una altitud de 3250 msnm.

El rastrojo de maca fue colectado en el distrito de Junín (meseta de Bombón a 4100 msnm), luego del proceso de la trilla mecánica para la obtención de semilla botánica del ecotipo amarillo. Del rastrojo colectado se tomaron 4 muestras de $100 \mathrm{~g}$, las que fueron molidas y mezcladas, tomando $10 \mathrm{~g}$ de cada una para los análisis químicos y el resto fue utilizado en los ensayos de digestibilidad.

La composición química proximal del rastrojo de maca se determinó empleando protocolos de la AOAC (1990). Para el ensayo de digestibilidad, cálculo del contenido de nutrientes digestibles totales y consumo voluntario se utilizó el procedimiento sugerido por Castro y Chirinos (2007).

La digestibilidad in vivo supone determinar la cantidad de material que no se degrada ni se absorbe mientras pasa a través del tracto digestivo, definiendo a la digestibilidad como el porcentaje de nutrientes digeridos y absorbidos (Lammers et al., 2009). Es así que se cuantificó la cantidad de nutrientes ingeridos y las cantidades que aparecen en las heces, para lo cual los cuyes fueron dispuestos en jaulas metabólicas que disponían de comederos y bebederos, pero que, además, permitieron colectar las heces y orina por separado (Castro y Chirinos, 2007).

En los ensayos de digestibilidad y consumo voluntario se utilizaron cuatro cuyes machos de la raza Wanka de 4 meses y peso promedio de $663 \mathrm{~g}$. El ensayo de digestibilidad consideró una fase pre-experimental donde los cuyes fueron alimentados con rye grass italiano (300 g/cuy/día), reemplazándolo gradualmente por el rastrojo de maca, de manera que el cambio fue total al séptimo día. Diariamente se retiraron $50 \mathrm{~g}$ del rye gras y se incluyeron $10 \mathrm{~g}$ de rastrojo de maca cada dos días, de manera que al día 7 solamente reci- 
bían $30 \mathrm{~g}$ de rastrojo de maca y agua de bebida, dejándolos con este alimento por tres días más para estabilizar su consumo. La fase experimental tuvo una duración de 8 días, donde los primeros siete fueron de control de ingesta y del segundo al octavo día se realizó la colección fecal (considerando que las heces colectadas en un determinado día corresponden a la alimentación del día anterior).

Las heces colectadas fueron pesadas en fresco e inmediatamente llevadas a la estufa para la determinación del contenido de materia seca (MS) y ser almacenadas hasta completar la fase experimental. Al final de la fase de campo, las heces fueron molidas y sometidas al análisis proximal (AOAC, 1990), empleándose una estufa para determinar el contenido de materia seca, un equipo Micro Kjeldahl para la determinación del $\mathrm{N}$ y transformar a proteína bruta con el factor 6.25, un equipo de extracción Soxhlet para la determinación de grasa y digestión ácida y alcalina para determinar fibra bruta. La ceniza se obtuvo por incineración en un horno-mufla. Para registrar el peso del alimento ofrecido y rechazado y las heces se utilizó una balanza electrónica de capacidad $1.2 \mathrm{~kg}$ con precisión de $0.1 \mathrm{~g}$.

El consumo voluntario fue determinado empleando la información generada durante la fase experimental por diferencia entre la cantidad ofrecida y el correspondiente residuo. La ingesta de proteína se determinó considerando la ingesta diaria de rastrojo y su aporte proteico resultado del correspondiente análisis proximal.

\section{Resultados y Discusión}

La composición química proximal del rastrojo de maca se muestra en el Cuadro 1. El rastrojo de la trilla de la semilla de maca es un producto bastante seco, de allí que su contenido de MS (88.7\%) es similar al de los henos y subproductos agrícolas secos, como el caso de la paja de avena $(90.1 \%)$, de la paja de cebada (88.2\%), panca de maíz $(87.2 \%)$ y paja de trigo $(90.1 \%)$, entre otros (Castro y Chirinos, 1997). Este bajo contenido de humedad del rastrojo de maca obligó a utilizar bebederos en el ensayo de digestibilidad para que los animales puedan suplir su requerimiento hídrico.

El 6.97\% de proteína total (PT) fue más alto que los reportados para la paja de avena (4.4\%), paja de cebada (4.1\%), panca de maíz (5.9\%), paja de trigo (3.6\%) (Castro y Chirinos, 1997), Puya llatensis (2.32\%) (Clemente et al., 2003) y paja de avena (4.9\%) (Torres et al., 2009), de manera que no solo es una fuente de volumen o fibra sino que también aporta proteína a la dieta. Contenidos de PT ligeramente mayores se reportan en los residuos de quinua (7.8\%) (Calcina, 2015) y follaje de camote nativo (12.5\%) (Sologuren, 2008).

El $36.35 \%$ de fibra cruda del rastrojo de maca (fue inferior al de la paja de cebada 42.4\%, paja de trigo 41.5\% (Castro y Chirinos, 1997), paja de avena $51.8 \%$ (Torres et al., 2009); y similar al contenido de fibra de la panca de maíz 35.1\% (Castro y Chirinos, 1997) y mayor al del follaje de camote nativo $24.1 \%$ (Sologuren, 2008). La característica más relevante del rastrojo de la trilla de maca ha sido su alto nivel de fibra, siendo apta para el uso en la alimentación de herbívoros. Además, se debe tener en cuenta el aporte de una serie de fitoquímicos de importancia nutricional y amplia acción farmacológica $(\mathrm{Li}$ et al., 2001; Dini et al., 2002; Bianchi, 2003; Gonzales et al., 2005; Gonzales, 2006; Castañeda et al., 2010; Gonzales, 2012; Esparza et al., 2015).

La digestibilidad de la MS seca del rastrojo de maca fue de $74.79 \%$ (Cuadro 2); valor similar al $73.3 \%$ en hojas de Phalaris tuberoarundinacea, $76.7 \%$ en totora sin inflorescencia, $77.6 \%$ en cáscara de zanahoria, $72.2 \%$ en cáscara de habas (Castro y Chirinos, 1997) y $76.0 \%$ en Puya llatensis (Clemente et al., 2003). Para el sub produc- 
to de trigo (SPT), cebada grano y maíz chala se reportan valores de 70.0, 83.0 y $59.0 \%$, respectivamente (Correa et al., 1994), 49\% para el kikuyo (Lagos et al., 2006), 81.4\% en los residuos de quinua «Jipi» (Calcina, 2015), $67.8 \%$ para el follaje de camote nativo cortado a los 60 días de edad (Sologuren, 2008), 70.6 y $69.2 \%$ para la cáscara de algodón y cascarilla de arroz (Garay et al., 2008) y $66.3 \%$ para el forraje seco de mucuna (Stizolobium deeringianum) (Valenzuela, 2015).

El 68\% de digestibilidad de la proteína del rastrojo de maca fue similar al registrado para la col lisa (69.0\%), maíz chala $(66.2 \%)$, cáscara de kiwicha $(64.3 \%)$ y cáscara de tarwi (65.6\%), siendo mayor que los reportados para el afrecho $(56.7 \%)$, cáscara de papa (55.6\%) y cáscara de zanahoria $(52.0 \%)$ (Castro y Chirinos, 1997) y follaje de camote nativo (56\% (Sologuren, 2008); resultados que indican que la proteína del rastrojo de maca es de alta digestibilidad para los cuyes.

El contenido de nutrientes digestibles totales (NDT) del rastrojo de maca fue de $71.21 \%$, valor que lo clasifica como un ingrediente energéticamente bueno. Este valor es similar a los calculados para la hoja de camote $(74.0 \%)$, hojas de Phalaris tuberoarundinacea $(70.3 \%)$, cáscaras de habas (70.1) y maíz amarillo (71.4\%). Por otro lado, el contenido de NDT fue más alto que aquellos para insumos fibrosos como las hojas de maíz chala (54.1\%), tallo del maíz chala (60.2\%), afrecho de morón (66.7\%) y cáscara de kiwicha (43.5\%), entre otros (Castro y Chirinos, 1997). Por su parte, Correa et al (1994), para los SPT, cebada grano y maíz chala se reportan contenidos de energía digestible de 3219,3271 y $2381 \mathrm{kcal} / \mathrm{kg}$, los que tendrían equivalentes de NDT de 73.1, 74.3 y $54.1 \%$, respectivamente. Asimismo, Zevallos et al. (1994) reportan 70.3\% de NDT para la hoja de morera, y Clemente et al. (2003) reportan $68.7 \%$ de NDT para Puya llatensis. Por su parte, Calcina (2015) re- porta $76 \%$ de NDT para los residuos de quinua $«$ Jipi».

Los cuyes consumieron el rastrojo de maca sin inconvenientes. El consumo del rastrojo fresco fue de $21.46 \mathrm{~g} / \mathrm{cuy} /$ día, que representa el $3.25 \%$ del peso vivo. Este consumo es similar a los registrados con cebada grano (3.9\%), hechizo de cebada $(3.78 \%)$ y afrecho de morón (3.04\%) (Castro y Chirinos, 1997). El consumo de MS/cuy/día fue de $19.04 \mathrm{~g}$, que representa el $2.89 \%$ del peso vivo; valor que se encuentra por debajo de los consumos registrados para el caso de panca de maíz (5.90\%), cebada grano $(3.45 \%)$, maíz amarillo (3.16\%), hechizo de cebada (3.36\%), cáscara de papa $(6.66 \%)$ y cáscara de zanahoria $(4.56 \%$ ) (Castro y Chirinos, 1997).

El consumo promedio de materia seca del rastrojo de maca por kilogramo de peso metabólico (Cuadro 3) se encuentra por debajo de los determinados para pastos cultivados como alfalfa, rye grass, phalaris, chala, col, totora y trébol, cuyo rango está entre 295 y $452 \mathrm{~g} / \mathrm{kg}^{0.75}$; sin embargo fue más alto que los reportados para subproductos agroindustriales como la cáscara de arvejas (117.9 g), cáscara de habas $(123.0 \mathrm{~g})$, afrecho de morón (138.1 g) y afrechillo de trigo (133.2 g) (Castro y Chirinos, 1997). Estos resultados indican que la el rastrojo de maca puede ser incluido en la dieta para cuyes.

En el Cuadro 4 se muestra la ingesta diaria (en gramos) de proteína, grasa, fibra cruda, ELN, ceniza y NDT. La ingesta promedio de proteína por kilogramo de cuy vivo fue $2 \mathrm{~g}$ /día. Según la NRC (1995), la necesidad de proteína de los cuyes depende de la fuente de nitrógeno en la dieta. Cuando la caseína era la única fuente mostraban un requerimiento inusualmente alto (30\%), disminuyendo cuanto mejor era el balance de aminoácidos (arginina, cistina, glicina, metionina). El porcentaje de proteína requerida disminuye drásticamente al utilizar fuentes proteicas como harina de soya. 
Cuadro 1. Composición química proximal (\%) del rastrojo de maca

\begin{tabular}{cccccccc}
\hline Muestra & $\begin{array}{c}\text { Humedad } \\
(\%)\end{array}$ & $\begin{array}{c}\text { Materia } \\
\text { seca } \\
(\%)\end{array}$ & $\begin{array}{c}\text { Proteína } \\
\text { total } \\
(\%)\end{array}$ & $\begin{array}{c}\text { Extracto } \\
\text { etéreo } \\
(\%)\end{array}$ & $\begin{array}{c}\text { Fibra } \\
\text { cruda } \\
(\%)\end{array}$ & $\begin{array}{c}\text { ELN }^{1} \\
(\%)\end{array}$ & $\begin{array}{c}\text { Ceniza } \\
(\%)\end{array}$ \\
\hline 1 & 12.65 & 87.35 & 7.74 & 4.12 & 36.04 & 39.96 & 11.14 \\
2 & 8.15 & 91.85 & 8.45 & 3.67 & 37.00 & 41.02 & 9.86 \\
3 & 11.56 & 88.44 & 7.29 & 3.00 & 34.56 & 48.94 & 6.21 \\
4 & 12.84 & 87.16 & 4.38 & 3.51 & 37.78 & 48.21 & 6.12 \\
\hline Promedio & 11.30 & 88.70 & 6.97 & 3.58 & 36.35 & 44.53 & 8.58 \\
\hline
\end{tabular}

${ }^{1}$ Extracto libre de nitrógeno

Cuadro 2. Coeficientes de digestibilidad del rastrojo de maca en cuyes

\begin{tabular}{cccccccc}
\hline Cuy & $\begin{array}{c}\text { Materia } \\
\text { seca } \\
(\%)\end{array}$ & $\begin{array}{c}\text { Proteína } \\
\text { total } \\
(\%)\end{array}$ & $\begin{array}{c}\text { Extracto } \\
\text { etéreo } \\
(\%)\end{array}$ & $\begin{array}{c}\text { Fibra } \\
\text { cruda } \\
(\%)\end{array}$ & $\begin{array}{c}\text { ELN }^{1} \\
(\%)\end{array}$ & $\begin{array}{c}\text { Materia } \\
\text { orgánica } \\
(\%)\end{array}$ & $\begin{array}{c}\text { NDT }^{2} \\
(\%)\end{array}$ \\
\hline 1 & 75.47 & 69.39 & 76.80 & 72.53 & 77.83 & 75.04 & 72.03 \\
2 & 75.87 & 66.54 & 76.24 & 72.55 & 79.76 & 75.75 & 72.66 \\
3 & 73.66 & 68.01 & 72.96 & 70.29 & 75.24 & 72.63 & 69.66 \\
4 & 74.15 & 68.08 & 75.06 & 71.77 & 75.48 & 73.42 & 70.47 \\
\hline Promedio & 74.79 & 68.00 & 75.27 & 71.78 & 77.08 & 74.21 & 71.21 \\
\hline
\end{tabular}

${ }^{1}$ Extracto libre de nitrógeno

${ }^{2}$ Nutrientes digestibles totales

Cuadro 3. Consumo voluntario en fresco y seco del rastrojo de maca en cuyes

\begin{tabular}{|c|c|c|c|c|c|c|}
\hline Cuy & $\begin{array}{l}\text { Peso vivo } \\
\text { (g) }\end{array}$ & $\begin{array}{c}\mathrm{CFV}^{1} \\
\text { (g/cuy/día) }\end{array}$ & $\begin{array}{c}\mathrm{CMS}^{2} \\
\text { (g/cuy/día) }\end{array}$ & $\begin{array}{l}\text { CFV } \\
\text { (\% del } \\
\text { PV) }\end{array}$ & $\begin{array}{l}\text { CMS } \\
(\% \text { del } \\
\text { PV) }\end{array}$ & $\begin{array}{c}\text { CMS } \\
\left(\mathrm{g} / \mathrm{kg}^{0.75}\right)\end{array}$ \\
\hline 1 & 615 & 20.70 & 18.36 & 3.37 & 2.99 & 148.67 \\
\hline 2 & 580 & 19.41 & 17.22 & 3.35 & 2.97 & 145.70 \\
\hline 3 & 780 & 23.36 & 20.72 & 2.99 & 2.66 & 140.37 \\
\hline 4 & 676 & 22.37 & 19.84 & 3.31 & 2.94 & 149.68 \\
\hline Promedio & 662.75 & 21.46 & 19.04 & 3.25 & 2.89 & 146.11 \\
\hline
\end{tabular}


Cuadro 4. Consumo de materia seca, proteína cruda, grasa, fibra cruda, extracto libre de nitrógeno (ELN), ceniza y nutrientes digestibles totales (NDT) por cuy/día (g)

\begin{tabular}{cccccccc}
\hline Cuy & $\begin{array}{c}\text { Materia } \\
\text { seca }\end{array}$ & $\begin{array}{c}\text { Proteína } \\
\text { total }\end{array}$ & $\begin{array}{c}\text { Extracto } \\
\text { etéreo }\end{array}$ & $\begin{array}{c}\text { Fibra } \\
\text { cruda }\end{array}$ & ELN & Ceniza & NDT \\
\hline 1 & 18.36 & 1.28 & 0.66 & 6.67 & 8.18 & 1.58 & 13.07 \\
2 & 17.22 & 1.20 & 0.62 & 6.26 & 7.67 & 1.48 & 12.26 \\
3 & 20.72 & 1.44 & 0.74 & 7.53 & 9.23 & 1.78 & 14.75 \\
4 & 19.84 & 1.38 & 0.71 & 7.21 & 8.83 & 1.70 & 14.13 \\
\hline Promedio & 19.04 & 1.33 & 0.68 & 6.92 & 8.48 & 1.64 & 13.55 \\
\hline Por kg de cuy & 28.73 & 2.01 & 1.03 & 10.44 & 12.80 & 2.47 & 20.45 \\
\hline
\end{tabular}

\section{Conclusiones}

- El rastrojo de maca proveniente de la trilla para la obtención de semilla botánica aporta mayor contenido de proteína y energía que otros residuos agrícolas.

- Los coeficientes de digestibilidad de la proteína, grasa, fibra, ELN y materia orgánica del rastrojo de maca están entre 68 y $77 \%$ y el contenido de NDT supera el $70 \%$.

- La ingesta del rastrojo de maca representa el $2.9 \%$ del peso vivo en base seca, con un equivalente de 146 gramos de materia seca por kilogramo de peso metabólico, aportando $2 \mathrm{~g}$ /día de proteína por kilogramo de cuy vivo.

- El rastrojo de maca puede ser utilizado en la alimentación de cuyes.

\section{Literatura Citada}

1. Alzamora L, Alvarez E, Torres D, Solís H, Colona E, Quispe J, Chanco M. 2007. Efecto de cuatro ecotipos de Lepidium peruvianum Chacón sobre la producción de óxido nítrico in vitro. Rev Perú Biol 13: 215-217. doi: 10.15381/ rpb.v13i3.2342
2. AOAC. 1990. Official Methods of Analysis of the Association of Official Analytical Chemists. Vol 1. 15 $5^{\text {th }} \mathrm{ed}$. Washington DC: AOAC. 1298 p.

3. [BCRP] Banco Central de Reserva del Perú. 2013. Informe económico y social de la Región Junín. [Internet]. Disponible en: http://www.bcrp.gob.pe/docs/ Proyeccion-Institucional/EncuentrosRegionales/2013/junin/ies-junin-2013.pdf

4. Bianchi A. 2003. Maca Lepidium meyenii. Bol Latinoam Plantas Med Arom 2(3): 26-44.

5. Calcina CG. 2015. Digestibilidad y valor energético de residuos de quinua «Jipi» en cuyes». Tesis de Médico Veterinario Zootecnista. Puno, Perú: Univ. Nacional del Altiplano. $76 \mathrm{p}$.

6. Camilonga C, Flores $\boldsymbol{U}$. 2003. Uso de maca (Lepidium meyenii) en cuyes ( $\mathrm{Ca}$ via porcellus) con baja fertilidad. En: XXVI Reunión Anual de la APPA. Pucallpa.

7. Castañeda B, Loja B, Puebla P, Gamarra F, Alvarado A, Muñoz AM, Enriques VY, Ibáñez L. 2010. Estudio botánico y fitoquímico de las hojas secas de maca de la meseta de Bombón, Junín-Perú. Rev Horizonte Médico 10: 13-22. 
8. Castro J, Chirinos D. 1997. Nutrición y alimentación de cuyes. Huancayo, Perú: Univ. Nacional del Centro del Perú. $263 \mathrm{p}$.

9. Castro J, Chirinos D. 2007. Nutrición animal. $2^{\circ}$ ed. Huancayo: Industria Gráfica. $263 \mathrm{p}$.

10. Castro J, Chirinos D. 2017. Nutrición Animal. $3^{\circ} \mathrm{ed}$. Huancayo, Perú: Gráfica José Impresores. 265 p.

11. Clemente JE, Arbaiza FT, Carcelén CF, Lucas AO, Bazán RV. 2003. Evaluación del valor nutricional de la Puya llatensis en la alimentación del cuy $(\mathrm{Ca}$ via porcellus). Rev Inv Vet Perú 14(1): 1-6. doi: 10.15381/rivep.v14i1.1583

12. Correa H, Hidalgo V, Vergara V, Montes T. 1994. Determinación de la digestibilidad de insumos energéticos proteicos y fibrosos en cuyes. En: XVII Reunión Anual de la APPA. Lima.

13. Dini A, Migliuolo G, Ratrelli L, Saturnino P, Schettino O. 1994. Chemical composition of Lepidium meyenii. Food Chem 49: 347-349. doi: 10.1016/03088146(94)90003-5

14. Dini I, Tenore C, Dini A. 2002. Glucosinolates from maca (Lepidium meyenii). Biochem Syst Ecol 30: 10871090.

15. Esparza E, Hadzich A, Kofer W, Mithöfer A, Cosio EG 2015. Bioactive maca (Lepidium meyenii) alkamides are a result of traditional Andean postharvest drying practices. Phytochemistry 116 : 138-48. doi: 10.1016/j.phytochem.2015.02.030

16. Garay IG, Vergara VR, Chauca LF, Remigio RME. 2008. Digestibilidad y energía digestible de la cáscara de algodón y cascarilla de arroz en cuyes (Cavia porcellus). Tesis de Ing. Zootecnista. Lima: Univ. Nacional Agraria La Molina. 63 p.

17. Gonzales C, Rubio J, Gasco M, Nieto J, Yucra S, Gonzales G. 2006. Effect of short-term and long-term treatments with three ecotypes of Lepidium meyenii (MACA) on spermatogenesis in rats. J Ethnopharmacol 103: 448-454. doi: 10.1016/j.jep.2005.08.035
18. Gonzales G, Miranda S, Nieto J, Fernández G, Yucra S, Rubio J, Yi P, Gasco M. 2005. Red maca (Lepidium meyenii) reduced prostate size in rats. Reprod Biol Endocrinol 3(1): 5. doi: 10.1186/1477-7827-3-5

19. Gonzales GF. 2006. Biological effects of Lepidium meyenii, maca, a plant from the highlands of Peru. In: Singh VK, Bhardwaj R, Govil JN, Sharma RK (eds). Natural Products. Series: Recent Progress in Medicinal Plants. USA: Studium Press 15: 217-242.

20. Gonzales GF. 2012. Ethnobiology and Ethnopharmacology of Lepidium meyenii (maca), a plant from the Peruvian Highlands. Evid Based ComplementAlternat Med 2012: 193496. doi: 10.1155/2012/193496

21. Lammers PJ, Carlson SL, Zdorkowski GA, Honeyman MS. 2009. Reducing food insecurity in developing countries through meat production: the potential of guinea pig (Cavia porcellus). Renew Agric Food Syst 24: 155-162.

22. Lagos BL, Velasco BA, Apráez GE. 2006. Digestibilidad de forrajes en cuyes Cavia porcellus mediante la técnica in situ. Rev Cienc Agríc 23: 136-145

23. Li G, Ammermann U, Quirós C. 2001. Glucosinolate contents in maca (lepidium Peruvianum Chacón) seeds, sprouts, mature plants and several derived commercial products. Economic Botany 55: 255-262.

24. Muhammad I, Zhao J, Dunbar C, Khan I. 2002. Constituents of Lepidium meyenii (maca). Phytochemistry 59: 105-110. doi: 10.1016/S0031-9422(01)00395-8

25. NRC. 1995. Nutrient requirements of laboratory animals. $4^{\text {th }}$ rev ed. Washington DC: Subcommittee on Laboratory Animal Nutrition. National Research Council. National Academy Press.

26. Perú Biodiverso. 2011. La cadena de valor de maca en la Meseta del Bombón. Análisis y lineamientos estratégicos. Ministerio de Comercio Exterior-GTZ. 
[Internet]. Disponible en: http:// bibliotecavirtual.minam.gob.pe/biam/ bitstream/handle/minam/1432/ BIV01208.pdf?sequence $=1$ \&isAllowed $=\mathrm{y}$

27. Shimada MA. 2007. Nutrición animal. México: Ed Trillas. 397 p.

28. Sologuren VJ. 2008. Producción y calidad nutricional del follaje de camote nativo (Ipomoea batata), para alimentación de cuyes, bajo condiciones de selva alta - Satipo. Tesis de Ingeniería en Ciencias Agrarias. Huancayo, Perú: Univ. Nacional del Centro del Perú. 101 p.

29. Torres GG, Arbaiza FT, Carcelén CF, Lucas AO. 2009. Comparación de las técnicas in situ, in vitro y enzimática (celulasa) para estimar la digestibilidad de forrajes en ovinos. Rev Inv Vet Perú 20: 5-9. doi: 10.15381/rivep.v20i1.348

30. Valenzuela RR. 2015. Determinación de la digestibilidad y energía digestible del forraje seco de mucuna (Stizolobium deeringianum) en cuyes. Tesis de Ingeniero Zootecnista. Lima: Univ. Nacional Agraria La Molina. Disponible en: $70 \mathrm{p}$.

31. Zevallos L, Hidalgo V, Moreno A, Montes T. 1994. Evaluación biológica de la hoja de morera mediante pruebas de digestibilidad y crecimientos en cuyes. En XVII Reunión Científica de la Asociación Peruana de Producción Animal. Lima. 